\title{
Filigrane
}

Écoutes psychothérapiques

\section{La psychanalyse plurielle. Notule pour une pratique sur mesure et dans les règles de l'art}

\section{Nathalie Zilkha}

Volume 17, numéro 1, printemps 2008

L’avenir du clinicien I

URI : https://id.erudit.org/iderudit/018788ar

DOI : https://doi.org/10.7202/018788ar

Aller au sommaire du numéro

Éditeur(s)

Revue Santé mentale au Québec

ISSN

1192-1412 (imprimé)

1911-4656 (numérique)

Découvrir la revue

Citer cet article

Zilkha, N. (2008). La psychanalyse plurielle. Notule pour une pratique sur mesure et dans les règles de l'art. Filigrane, 17(1), 44-50.

https://doi.org/10.7202/018788ar
Résumé de l'article

À la question de savoir comment l'analyse peut répondre à la contrainte sociale qui refuse les limites et l'endeuillement, l'auteur cherche des réponses dans la complexité de la pensée analytique et dans la diversité des démarches analytiques. Se pose inévitablement aussi la question de la résistance des cliniciens eux-mêmes en lien avec les avatars de leur travail personnel, notamment la résistance liée à l'idéalisation de l'analyse ou d'une pensée analytique. Celle-ci ne manque pas de figer la pratique et la pensée psychanalytiques. 


\title{
La psychanalyse plurielle. Notule pour une pratique sur mesure et dans les règles de l'art
}

\author{
nathalie zilkha
}

\begin{abstract}
À la question de savoir comment l'analyse peut répondre à la contrainte sociale qui refuse les limites et l'endeuillement, l'auteur cherche des réponses dans la complexité de la pensée analytique et dans la diversité des démarches analytiques. Se pose inévitablement aussi la question de la résistance des cliniciens eux-mêmes en lien avec les avatars de leur travail personnel, notamment la résistance liée à l'idéalisation de l'analyse ou d'une pensée analytique. Celle-ci ne manque pas de figer la pratique et la pensée psychanalytiques.
\end{abstract}

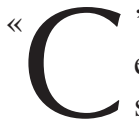

'est vous le chirurgien esthétique? On m'a dit que c'était au premier étage », me demande récemment une femme qui sonne à ma porte. Ce scénario insolite se répète plusieurs fois au cours de la même semaine avant que n'apparaisse sur la porte adjacente de mon cabinet une plaque portant l'inscription Anti Aging. Pour la plupart, mes analysants ont réagi avec humour: «Qu'est-ce qu'une psychanalyste fait à côté d'un institut anti-aging?» mais une pointe de regret était parfois perceptible. Après tout, que font-ils à souffrir trois ou quatre fois par semaine pendant des années pour se reconnaître et s'accepter ainsi que pour admettre la finitude de la vie et le temps qui passe, alors que tout pourrait être réglé par un «simple» lifting, une injection de botox ou une consultation de re-looking? Il est vrai que pour tout rajeunissement nous proposons... l'exploration de l'infantile et son endeuillement.

Et que dire de nos patients qui, lorsqu'ils consultent Internet, apprennent que les difficultés qu'ils présentent peuvent être «classés » en un trouble de la personnalité pour lequel d'aucuns préconisent un traitement médicamenteux (à vie).... ?

Ces épisodes sont illustratifs de ce qui peut se passer du côté des patients. Du côté des soignants, la situation n'est pas très différente; d'autres formations «présentent mieux », sont plus accessibles, plus facilement «maîtrisables», ou, pour reprendre un concept d'actualité, nettement plus user-friendly.

\section{La pratique analytique aurait-elle pris des rides?}

Le thème auquel les rédacteurs de la revue nous proposent de réfléchir est délicat. Quelles caractéristiques actuelles, du monde extérieur et de la réalité psychique individuelle et groupale, viennent-elles bousculer la pratique analytique 
et que pouvons-nous en inférer pour notre avenir de cliniciens ? Conjointement aux diverses réflexions basées sur notre expérience de clinicien, ce sujet ne manque pas de mobiliser, en chacun, des mouvements affectifs dont certains sont inconscients. Au-delà de nos inévitables inquiétudes concernant notre avenir, je pense surtout, et c'est autrement plus difficile à reconnaître, à nos résistances face à la psychanalyse, voire à la forme de résistance bien particulière qu'est l'idéalisation de l'analyse ou de l'institution analytique. Ce sont autant de traces de mouvements transférentiels et de témoignages de notre relation à notre propre réalité psychique qui sous-tendent notre investissement de l'analyse et, par conséquent, notre vue de celle-ci et de son avenir.

La situation peut paraître inquiétante et pourtant, sans pour autant nous mettre des œillères sur ce qui se passe chez les voisins, sur les terrains thérapeutiques proches ou plus lointains, l'avenir de notre pratique se trouve dans la spécificité de l'écoute de la réalité psychique qu'elle permet et cela, suivant le titre du colloque de la Société psychanalytique de Paris rappelé par le comité de rédaction, dans la diversité de la pratique. L'avenir du clinicien n'engagerait-il pas, en effet, une psychanalyse tout à la fois rigoureuse dans ses références et dans son modèle de théorisation et plurielle dans ses dispositifs?

Nous ne prendrions pas trop de risque à dire que la psychanalyse est déjà plurielle, de fait, de par ses enracinements théoriques diverses ou encore en raison de (ou grâce à) ses différentes pratiques : dispositif d'analyse classique, de face à face, de psychodrame, de groupe, entretiens avec un clinicien. Par ailleurs, cette pratique différentielle et les ajustements qui lui sont corrélatives ne concernent pas seulement le cadre de travail mais se jouent dans la séance même ; pensons notamment au choix et à la forme des diverses interprétations proposées par l'analyste.

\section{La tentation de l'idéalisation, son coût et l'effet de sa chute}

Dans son argument, le comité de rédaction souligne le poids de l'idéalisation préalable de l'analyse et son prix, d'autant qu'elle a été suivie d'une chute de celleci. Il précise aussi que les analystes ont peut-être eux-mêmes participé à cette idéalisation. De quoi est-elle faite? Comment se manifeste-t-elle? Que pouvonsnous lui préférer ou lui opposer?

Avant de m'atteler à ces questions je voudrais souligner que nous ne sommes pas les seuls à reconnaître de tels changements. Récemment, des médecins spécialistes et chefs de service s'exprimaient ainsi dans un éditorial :

«Aucune profession libérale n'a subi une régression similaire à celle de la médecine au cours des vingt dernières années ! Non pas en termes de performance, mais de reconnaissance sociale. [...] Du mandarinat d'autrefois, nous avons basculé dans l'asservissement bureaucratique à des dirigeants non médecins, éloignés de la réalité du terrain. À tel point que bon nombre de nos amis s'étonnent de la perte d'aura de notre profession, alors qu'ils 
restent admiratifs de ses succès!» (Philippe Monnier et JeanPhilippe Guyot, Revue médicale suisse du 3 oct.-07, p. 2195.)

Mais revenons à la pratique qui est la nôtre. Je me souviens de discussions inquiètes avec des collègues au début de ma formation. «Autant d'analystes, autant d'interprétations différentes », constations-nous avec dépit, comme si, idéalement, il ne devait y avoir qu'une seule perspective, une interprétation unique du matériel clinique, nécessairement «la bonne», à laquelle seraient opposées toutes les autres, «les mauvaises ». Ou encore nous était-il peut-être difficile de concilier la pluralité des perspectives que nous avions à intégrer avec l'investissement électif que chaque analyste semblait faire de son point de vue ou de celle de son école. Le paradoxe était contraignant. N'y aurait-il qu'une vérité et, si oui, laquelle ou, au contraire, y en aurait-il irrémédiablement toujours plusieurs, voire toutes et donc aucune?

Chacune de ces conceptions pouvait se révéler inquiétante pour les jeunes cliniciens que nous étions; de fait l'idéalisation de la pratique analytique ou l'appropriation idéologique d'une perspective pouvaient présenter des solutions au malaise que nous ressentions. Ce n'est que plus tard que nous avons pu véritablement estimé l'art avec lequel certains de nos aînés pouvaient discuter de la pertinence d'une interprétation ou de telle autre en appréciant ce qu'elle avait amené (ou aurait pu amener) et, corrélativement, ce qu'elle avait manqué (ou aurait pu manquer) de faire advenir. Ainsi était implicitement reconnu aussi qu'il ne pouvait jamais y avoir d'interprétation unique d'un matériel, à moins que celuici ne soit artificiellement simplifié.

Il est banal de dire qu'une autre tendance à l'idéalisation de l'analyse par le clinicien provient des vicissitudes de son analyse personnelle, voire de son inachèvement. Plus encore que l'inévitable «inachèvement» de la cure ou du travail analytique, ces malheureux inachèvements trouvent parfois la voie vers un investissement particulier de l'analyse ou de l'institution analytique. Dans ces situations par exemple, contrairement à l'analysant non clinicien qui «choisira» divers aspects de sa vie sur lesquels il pourra transférer la part de transfert non endeuillé, pour l'analysant clinicien, actuel ou futur, le déplacement du transfert sur l'analyse ou sur l'institution analytique est très (trop) facile, immédiat et peut, tant par sa forme que par son intensité, encourager l'idéalisation. D'autant que les divers groupes professionnels agissent comme des ferments solides pour ce type de mouvements psychiques.

À défaut de pouvoir approfondir ici ces divers points qui contribuent à la participation du clinicien à l'idéalisation de l'analyse ou du travail analytique, je voudrais simplement signaler un élément de la conjonction singulière de ce type de travail qui renforce aussi la tendance à l'idéalisation : à l'intense investissement dont le clinicien est l'objet de la part de ses patients par le biais du transfert se juxtapose la douloureuse réalité de ses limites et de celles de son cadre.

Dit autrement, une grande alliée de l'idéalisation est la croyance en un dispositif unique ou en une vérité unique et son interprétation, tant de la part du 
clinicien que de son patient (on est du côté de l'idéologie ou de la fétichisation de l'interprétation, voire du culte de la personnalité de l'interprétant); plus la pensée est unique, plus on se rapproche d'une idéologie militante qui appelle à l'idéalisation de la personne ou de la méthode. A contrario, tant qu'elles s'appuient sur une théorisation rigoureuse et qu'elles ne sont pas elles-mêmes fétichisées, la pluralité et la complexité s'opposent à l'idéalisation.

Travaillons cette perspective dans la séance elle-même, au niveau de l'interprétation du matériel de séance.

\section{Une pluralité d'interprétations non antagonistes}

La pluralité des interprétations non antagonistes est explicite dès les premiers textes de Freud. Elle est corrélative du travail de condensation et de déplacement et témoigne de la complexité de l'appareil psychique et de son fonctionnement. Ceci évoque bien entendu la surdétermination que Laplanche et Pontalis (1967) définissent ainsi :

«Fait qu'une formation de l'inconscient — symptôme, rêve, etc. - renvoie à une pluralité de facteurs déterminants. Ceci peut être pris en deux sens assez différents :

a) La formation envisagée est la résultante de plusieurs causes, alors qu'une seule ne suffit pas à en rendre compte;

b) La formation renvoie à des éléments inconscients multiples, qui peuvent s'organiser en des séquences significatives différentes, dont chacune, à un certain niveau d'interprétation, possède sa cohérence propre. Ce deuxième sens est le plus généralement admis. » (p. 467)

Prenons pour premier exemple le célèbre rêve du saumon fumé, aussi appelé rêve de la Bouchère décrit dans L'interprétation des rêves (Freud 1900, 135). Freud explicite plusieurs interprétations possibles et en évoque implicitement d'autres. Il précise d'ailleurs que ces interprétations ne s'excluent pas. Après avoir évoqué une première interprétation, Freud précise encore:

«Ce même rêve comporte une autre interprétation plus délicate. [...] Les deux explications ne se contredisent pas, mais se recouvrent et sont un bel exemple du double sens que le rêve, comme toutes les autres structures psychopathologiques présente habituellement. » $(1900,135)$

Tournons-nous maintenant vers Dora et sa production de symptômes. Ainsi, lorsque Dora vient un jour à sa séance avec un symptôme nouveau, Freud lui demande «Qui copiez-vous là ?». Considérons ce passage : «Elle [Dora] se plaignit un jour d'un symptôme en apparence nouveau, de douleurs aiguës d'estomac, et 
lorsque je lui demandai: «Qui copiez-vous là?» je tombai juste.»(p. 26) Mais Freud ne s'en tient pas là. En effet pour trouver sa pertinence, le «qui copiez-vous » doit ouvrir à une réflexion sur ce qui motive cette identification. Freud poursuit:

«Elle avait rendu visite, la veille, à ses cousines.... La cadette s'était fiancée; l'aînée, à cette occasion s'était mise à souffrir de l'estomac...»

Il comprend ainsi que les maux d'estomac de Dora «témoignaient de ce qu'elle s'identifiait avec sa cousine qualifiée de simulatrice». Mais il ne suffit pas de dire cela, il faut en comprendre les raisons. Freud se demande si Dora envie celle qui était la plus heureuse ou si elle se retrouve dans celle qui vient de subir un chagrin d'amour. Tout cela est surdéterminé puisque cela renvoie aussi, par le biais du couple de $\mathrm{M}$. et $\mathrm{M}^{\mathrm{me}} \mathrm{K}$, au couple œdipien.

À la polysémie du rêve ou du symptôme correspond ici une polyphonie identificatoire, une autre manifestation de la richesse et de la complexité de notre réalité psychique et, de fait, une chance du travail analytique de permettre à ces productions psychiques de se déplier grâce au transfert et à l'écoute singulière de l'analyste.

Le troisième exemple que je voudrais donner concerne celui de l'articulation entre ce qui provient du monde extérieur et ce qui provient de la pulsionnalité propre du sujet. Il me semble, en effet, essentiel que nous déconstruisions l'opposition entre intérieur et extérieur pour sortir de l'idéologie. Pour cela je reprendrai un court passage de «Inhibition, symptôme et angoisse» (Freud, 1926), l'un des travaux de Freud dans lesquels cette question est traitée de manière explicite quoique complexe. Freud tente entre autres de différencier l'angoisse de réel et l'angoisse névrotique mais la tâche n'est pas simple. Si de prime abord les choses pouvaient paraître claires puisque :

«Le danger de réel est un danger que nous connaissons, l'angoisse de réel est l'angoisse devant un tel danger connu. L'angoisse névrotique est angoisse devant un danger que nous ne connaissons pas. Le danger névrotique doit donc être recherché d'abord; l'analyse nous a enseigné que c'est un danger de pulsion.» (p. 77)

Très vite cette distinction tombe, notamment lorsqu'il est question d'un travail analytique, d'autant que ces deux types d'angoisse peuvent se montrer mélangées. qui plus est c'est surtout de leur rencontre que naît leur efficience : la revendication pulsionnelle devient objet d'angoisse parce qu'un danger externe serait associé à sa satisfaction. D'autre part,

«Il faut aussi que le danger (de réel) externe ait subi une intériorisation pour pouvoir devenir significatif pour le moi [...]» (p. 80) 
Si pour interpréter, il faut bien faire un choix en appui sur le transfert et mettre davantage l'accent sur un sens plutôt qu'un autre, il n'en reste pas moins que de savoir qu'il y aurait d'autres sens, lectures ou interventions possibles est déterminant pour la qualité de notre travail. La méconnaissance de cette complexité conduirait à penser que la psychanalyse est «vieux jeu » ou obsolète.

Mais tout n'est pas possible, les sens ne sont pas équivalents et pertinents. Nos références théoriques sont essentielles. Je n'entends pas par là une théorie qui serait déjà là, toute faite et toute prête à être appliquée. Je suis davantage intéressée par le concept de processus de théorisation et à l'impératif de théorisation pour le travail clinique. Florence Quartier (2004) met ce thème au travail, dans un magnifique ouvrage, fruit de sa profonde expérience de psychiatre et de psychanalyste, Freud clinicien. Elle insiste sur l'importance du processus théorisation pour la démarche clinique et ce que nous perdons à vouloir le contourner. Démarche clinique et processus de théorisation sont consubstantiels. Je pense que c'est à cette articulation favorable de la clinique et du processus de théorisation qu'elle fait référence lorsqu'elle nous propose :

«J'espère qu'il vous semble plus clair que la démarche psychanalytique (ce qui n'est pas la même chose que l'application de la cure psychanalytique en bonne et due forme) peut être utile dans n'importe quelle situation, à tout moment et pour tous les patients, à condition bien sûr d'être utilisée à bon escient et de manière tout à fait professionnelle. » $(2004,38)$

Cette position interne du clinicien permet à la psychanalyse de rester bien vivante tout en préservant la possibilité, lorsque les conditions sont réunies, d'un travail analytique spécifique. La portée de la psychanalyse s'en trouve élargie et renforcée.

Il serait utile d'approfondir les raisons de l'idéalisation de l'analyse qui influencent tant la position interne du clinicien face à la démarche psychanalytique et l'entravent dans sa pratique. Cette idéalisation est le reflet d'un travail personnel en souffrance, en impasse ou inachevé, ou encore la conséquence d'un noyau traumatique réactualisé par l'analyse. J'en suis venue à penser que dans ces cas-là les cliniciens d'orientation analytique pourraient être de terribles ennemis de l'analyse.

À l'instar de Dorian Gray qui, au moment de la chute de l'idéal, voit vieillir d'un coup le reflet qu'est son portrait, ne serait-ce pas la chute brutale de l'idéalisation de l'analyse par le clinicien qui fait prendre des rides à l'analyse ?

nathalie zilkha

5, ch. malombré

1206 genève suisse

nathalie.zilkha@bluewin.ch 
Filigrane, automne 2007

\section{Références}

Freud, S.,1900, L'interprétation des rêves, Paris, PUF, 1967.

Freud, S., 1905, Dora, in Les cinq psychanalyses, Paris, PUF.

Freud, S., 1926, Inhibition, symptôme et angoisse, ed Quadrige, PUF, 2005.

Laplanche, J. et Pontalis, J.-B., 1967, Vocabulaire de la psychanalyse, Paris, PUF.

Quartier, F., 2004, Freud clinicien. Pratiques cliniques contemporaines en psychiatrie et médecine, Paris, Doin. 